www.jmscr.igmpublication.org

Impact Factor 5.244

Index Copernicus Value: 83.27

ISSN (e)-2347-176x ISSN (p) 2455-0450

crossref DOI:_http://dx.doi.org/10.18535/jmscr/v4i8.43

\title{
Influence Of Implant Shape On Stress Distribution In The Maxilla And Mandible -A Review
}

\author{
Author \\ T.K.Vidhya \\ Third Year BDS, Saveetha Dental College And Hospitals \\ Email-vidhya.thirumalaisamy@gmail.com
}

\begin{abstract}
Influence of Implant Shape on Stress Distribution in the Maxilla and Mandible is a review article to analyse the stress distribution among the various shapes of implants and the bone response to the stress. The implant shapes are cylindrical, conical, screw. In cylindrical implant higher stress values are obtained under pure contact. Significant bone loss occurs when compact bone overloading occurs. The conical implant with additional retention gives less stress in axial loading at the neck of implants. The stepped implant has highest stress in the step and better structural stability whereas screw shape has greatest retention and initial stability. The lower maximum stress level in the stepped implant model is due to a low stiffness of the stepped implant. Screw implants provide greatest retention and initial stability and increases surface contact. Thus it shows that stress distribution occurs in different levels in different shapes of implant.
\end{abstract}

KEYWORDS: Stress distribution, implant shape, bone, Pontic, retention.

\section{INTRODUCTION}

Implants as a treatment options for replacement of missing teeth is gaining momentum among the people involved in the dental field as well as the community in the past few years. The success of a dental implant is directly affected by the stress distribution and maximum stress magnitude in the bone adjacent to the implant. The elastic modulus of the implant and the bone is different. To minimize these effects, implant geometry optimized to produce a more uniform stress pattern. Hence forces and moments transferred from implant to the surrounding bone depend on the type of loading, the bone-implant interface, implant geometry, and prosthesis type, and the quality and quantity of the surrounding bone.
Major contributions include implant length, design and diameter. Different implant shapes lead to significant variations in stress distributions in the bone. The combination of microscopic surface topography and macroscopic levels of implant design may be essential to create a stable bone implant interface in a low density bone. The object of this review article is to summarize the different implant design and its stress distribution in the bone implant interface.

\section{TYPES OF IMPLANT FORCES}

Favourable and unfavourable force distribution is key to design and selection of an implant based on the macroscopic features it may have. Misch identified three main types of load an implant may 
endure at the interface between the implant surface and bone. These three forces are compressive, tensile and shear (Figure 4-2). Compressive forces have been shown to be the most favourable when discussing bone possibly due in part to a concept developed by Wolff in 1892. Wolff observed a direct relationship with increasing mechanical loading and reactive bone formation. Tensile and shear forces are thought to be unfavourable due to an observed weakening of the bone. Efforts are therefore, focused on increasing compressive forces and minimizing the tensile and shearing forces which may weaken the bone to implant interface. , efforts to attain this have been made through tapering of the implant body and adjusting the thread design (Holmgren et al., 1998, Misch, 2008, Lemons, 1993).

\section{CYLINDRICAL IMPLANT}

Endosseous cylindrical implants were originally designed by an organization Known as the International Team for Implantology (ITI) beginning in 1974. The initial design was a hollow-cylinder which was thought to improve the surface area for increased bone-to-implant contact. Cylinder implants depends on coating or surface condition to provide microscopic retention to the bone., survival for hollow cylinder implants were less than ideal and are rarely seen available in the present implant market. Although endosseous cylindrical implants have shown greater success than the blade, pin and disk-like implants; the surface in contact with the bone under load is subject to heavy shearing forces and as a result rely heavily on the implant surface or microscopic characteristics of the implant. Cylinder implants are usually pushed or tapped in to a prepared bone site. Thread implants are primarily available in a parallel cylinder or tapered cylinder design. The amount of bone in contact with the implant did not significantly change, thus suggesting a role of implant design in protecting the bone from excessive stress and strain. Shortly after the development and use of the endosseous cylindrical implants a thread or screw shape was added to the body of the cylinder. Currently the most commonly used implant design available, the addition of a thread pattern allowed for implants to engage surrounding bone and achieve excellent initial stability following placement. Threaded cylindrical endosseous implants were parallel walled and have been shown to be successful over long periods of time. Using single-tooth implants, the results suggested the stresses were more evenly distributed in the tapered form rather than the strictly cylindrical. Cylindrical form implants with no thread or a thick squared thread were found to have the lowest compressive forces found at the bone crest. While implants with a tapered form and v-threads were found to have the highest compressive forces at the bone crest. Cylindrical implants were found to have less compressive force at the bone crest in compared to tapered form implants. Stress distributions within the bone and found implants with curvature such as the conical or stepped design introduced significantly higher stresses than the cylindrical or cylindrical with a thread pattern.

\section{STEP SHAPED IMPLANT}

The load transfer to bone is via the steps, the stress in the apical base is reduced but High stress peaks are seen below the steps. The notch effect shows high tensile stresses at the edge of steps. The compressive stress for pure contact is 11.0 MPa while it is $4.6 \mathrm{MPa}$ using fixed bond system. Tensile stresses are found to be of the same intensity. This shows more stress dissipation and improved loading of the crestal bone supporting of the alveolar bone from the root analogue shape compared to cylindrical or tapered implants. It offers better structural stability and it transfers stress more evenly to surrounding bone, thus preventing regions of high bone resorption next to regions of low bone activity. The stepped implant has highest stress in the step and better structural stability whereas screw shape has greatest retention and initial stability. The lower maximum stress level in the stepped implant model is due to a low stiffness of the stepped implant. 


\section{CONICAL IMPLANTS}

In a fixed bond system, the values of stress obtained in conical are similar to that of a cylindrical implant, however due to the smaller radii of curvature at the apex, the Stress values are estimated to be $2.8 \mathrm{MPa}$, which is twice as high as the cylindrical implant. The conical implant with additional retention gives less stress in axial loading at the neck of implants. Using single-tooth implants, the results suggested the stresses were more evenly distributed in the implants with a tapered form and v-threads were found to have the highest compressive forces at the bone creste tapered form rather than the strictly cylindrical (Holmgren et al., 1998).

\section{CONCLUSION}

Macroscopic and microscopic design features of dental implants play a role in initial and long term stability following placement. Due to force distribution through these design features and the variations seen in bone quality and quantity, there may not be a perfect implant design which would suit all needs and indications. Rather than the current trends with implant companies unifying their implant products surgically, based on the principles outlined, implant design principles could necessitate multiple macroscopic implant designs with more unified prosthetic platform.

\section{REFERENCE}

1. Abuhussein, H., Pagni, G., Rebaudi, A. \& Wang, H. L. (2010). The effect of thread pattern upon implant Osseo integration. Clinical oral implants research 21, 129136. doi:10.1111/j.1600-0501.2009.01800.x.

2. Albrektsson, T. (2003). The host-implant interface: biology. The International journal of prosthodontics 16 Suppl, 29-30; discussion 47-51.

3. Albrektsson, T., Branemark, P. I., Hansson, H. A. \& Lindstrom, J. (1981a). Osseo integrated titanium implants. Requirements for ensuring a long-lasting, direct bone-to-implant anchorage in man. Acta Orthop Scand 52, 155-170.

4. Albrektsson, T. \& Sennerby, L. (1991). State of the art in oral implants. Journal of clinical periodontology 18, 474-481.

5. Aljateeli, M. \& Wang, H. L. (2013). Implant Microdesigns and Their Impact on Osseo integration. Implant dentistry. doi:10.1097/ID.0b013e318278a90b 